\title{
Programas de Melhoria de Processo de Software - Uma pesquisa sobre a influência dos aspectos humanos
}

\author{
Davi Viana dos Santos ${ }^{1}$, Dalton Vilela ${ }^{2}$, Cleidson de Souza ${ }^{3}$, Tayana Conte ${ }^{1}$ \\ ${ }^{1}$ USES - Grupo de Pesquisa em Usabilidade e Engenharia de Software, Programa de \\ Pós-Graduação em Informática - Universidade Federal do Amazonas (UFAM) \\ Manaus - Amazonas \\ ${ }^{2}$ Fundação Centro de Análise, Pesquisa e Inovação Tecnológica (FUCAPI) \\ Manaus - Amazonas \\ ${ }^{3}$ IBM Research - Brazil \\ São Paulo - SP \\ \{davi.viana, tayana\}@dcc.ufam.edu.br, dalton.vilela@fucapi.br, \\ cleidson. desouza@acm.org
}

\begin{abstract}
Resumo. É sabido que diversos fatores podem influenciar o sucesso de um programa de melhoria de processo de software (MPS). Os resultados de pesquisas anteriores mostram que alguns destes fatores são de natureza humana e social. Por esta razão, é importante analisar os aspectos humanos envolvidos a fim de obter uma maior compreensão da influência destes em um programa MPS. Este artigo discute os principais resultados de uma pesquisa qualitativa conduzida em três diferentes fases para analisar quais fatores humanos tiveram maior influência do ponto de vista dos colaboradores das organizações. O objetivo é aprofundar a compreensão sobre como estes aspectos humanos podem influenciar um programa de MPS.
\end{abstract}

\begin{abstract}
It is widely known that several factors can influence the success of Software Process Improvement (SPI) programs. Results from previous research have shown that human and social aspects influence these programs in different ways. For this reason, it is important to analyze the human aspects involved on SPI programs to gain a better understanding about their influence on such programs. This paper discusses the major findings of a qualitative research conducted in three different phases to analyze which factors were most influential from the point of view of the professionals working in the organizations. Our ultimate goal is to deepen the understanding about how human aspects can influence a SPI program.
\end{abstract}

\section{Introdução}

Programas de melhoria de processo de software (MPS) são conduzidos visando diminuir o retrabalho e aumentar a produtividade das organizações desenvolvedoras de software (Rocha et al., 2005b), pois as mesmas partem da premissa que o aumento na qualidade do processo pode elevar a qualidade do produto final (Osterweil, 1987). Os resultados da pesquisa sobre a implementação do programa MPS.Br apresentados por Travassos e Kalinowski (2010) corroboram com as afirmativas apresentadas, uma vez que mostram indicadores de melhoria na qualidade do produto final e aumento da produtividade nas 
organizações brasileiras que foram avaliadas no modelo MPS. Esses indicadores se baseiam principalmente na quantidade dos pontos de função das organizações avaliadas.

Para conduzir programas de MPS, as organizações normalmente se baseiam em modelos de referência, como o MPS.BR (SOFTEX, 2009) e o CMMI (SEI, 2006). Esses modelos são fundamentados na tríade processo-tecnologia-pessoas (Sandhof, 2004). No elemento processo, têm-se os modelos de referência bem definidos que são largamente adotados pela indústria e estudos recentes indicam a satisfação das organizações em aplicar estes modelos em seus processos (Travassos e Kalinowski, 2010). O componente tecnologia é apoiado por ferramentas e ambientes de desenvolvimento de software, tais como a estação TABA (Rocha et al., 2005a) e o WebApsee (Sales et al., 2010). Em relação ao elemento pessoas, o número de pesquisas ainda está aquém do esperado, pois é necessária uma melhor compreensão do elemento humano que influencia os programas de MPS. Algumas pesquisas ressaltam que falhas em programas de MPS são de natureza humana e social, embora a área de engenharia de software seja vista como sendo de natureza técnica (Santos et al., 2011). Deste modo, pesquisas adicionais sobre a influência do elemento humano em programa de MPS são necessárias.

Contudo, analisar os aspectos humanos e sociais na Engenharia de Software não é uma tarefa trivial (Conte et al., 2009). É necessário utilizar abordagens que facilitem a identificação e compreensão satisfatória do objeto de estudo. Segundo Seaman (2008), os aspectos humanos podem ser mais bem compreendidos através da execução de pesquisas de caráter qualitativo. Estas investigações são conduzidas com a finalidade de obter um conhecimento intersubjetivo e compreensivo acerca de determinado fenômeno (Godoi et al., 2006). Além disso, pesquisas qualitativas permitem uma compreensão mais abrangente de todo o fenômeno em estudo, o que é necessário para analisar questões complexas da engenharia de software (Seaman, 1999).

Pesquisas recentes mostram a importância de estudos qualitativos para investigar os diversos fatores que podem influenciar os programas de MPS (Coleman e O'connor, 2008; Fernandes e Oliveira, 2010; Montoni e Rocha, 2010). Além da possibilidade de responder questões que envolvem variáveis difíceis de quantificar e auxiliar a responder o porquê de questões já abordadas nas pesquisas quantitativas, os métodos qualitativos possuem a vantagem de obrigar o pesquisador a se aprofundar na complexidade do problema ao invés de abstraí-lo. Desta forma, é possível ter resultados mais ricos e informativos (Seaman, 2008). Contudo, os resultados dependem do contexto no qual as organizações estão inseridas (Montoni e Rocha, 2010). Apesar desta limitação, os resultados dos estudos qualitativos permitem identificar comportamentos coerentes dos colaboradores das organizações, o que permite a construção de um "corpo de conhecimento" abrangente sobre programas de MPS e suas interações com aspectos humanos.

Com esta motivação, este artigo apresenta os resultados de uma pesquisa qualitativa com o objetivo de compreender quais aspectos humanos influenciam um programa de MPS, do ponto de vista dos colaboradores das organizações que foram avaliadas no nível G do MPS.BR. Desta forma, a questão de pesquisa que norteia este trabalho é a seguinte: Segundo os colaboradores, qual a influencia dos aspectos humanos em um programa de melhoria de processo de software? 
Espera-se que os resultados desta pesquisa permitam um entendimento mais rico sobre a influência dos aspectos humanos em programas de MPS. Desta forma, este trabalho auxilia implementações de MPS apresentando possíveis medidas que podem ser adotadas para evitar influências negativas relacionadas aos aspectos humanos. Este trabalho apresenta uma análise conjugada dos resultados de cada fase desta pesquisa. Resultados parciais são descritos em Matos et al. (2010) Santos et al. (2010) e Santos et al. (2011) e analisados com dados adicionais apresentados no presente artigo.

O restante deste artigo está estruturado da seguinte forma. A Seção 2 apresenta estudos anteriores que identificam diferentes aspectos que influenciam programas de MPS. A Seção 3 detalha como foi conduzida a pesquisa qualitativa descrita neste trabalho. A Seção 4 discorre sobre os resultados encontrados nas fases desta pesquisa. Por fim, a Seção 5 apresenta as principais conclusões deste trabalho e os trabalhos futuros a serem conduzidos.

\section{Identificação de influências em programas de MPS}

Os diversos fatores que podem influenciar o sucesso de um programa de MPS, bem como suas interações causas, efeitos e formas de tratamento são habitualmente denominados de Fatores Críticos de Sucesso (FCS). É crescente o número de estudos que abordam as questões técnicas e sociais envolvidas em programas de MPS, devido a relatos das dificuldades que as organizações enfrentam ao executar um programa de MPS (Montoni e Rocha, 2010). O propósito desses estudos é obter um melhor entendimento sobre essas questões. Por exemplo, Dyba (2000) descreve um estudo cujos resultados sugerem que a competência e o treinamento dos colaboradores da equipe e o monitoramento das atividades destes são questões chave para o sucesso de um programa de MPS. Posteriormente, em outro estudo, Dyba (2003) descreve que o tamanho da organização é um fator que pode influenciar no sucesso da implantação de programa de MPS, pois pequenas organizações, que obtiveram sucesso nos programas de melhoria, apresentaram um grande grau de participação dos colaboradores e uma maior aprendizagem da abordagem do programa de MPS.

Em relação aos fatores motivacionais, Baddoo e Hall (2002) descrevem um estudo abordando a identificação de questões que podem influenciar um programa de MPS do ponto de vista dos colaboradores de organizações do Reino Unido. Entre os fatores motivacionais encontrados, destacam-se: (1) o sentimento de dono da execução do processo, isto é, os colaboradores precisam se sentir responsáveis pelo processo no qual trabalham; (2) a visão dos benefícios que o MPS traz para a empresa apresentando as experiências positivas do MPS; (3) haver recursos suficientes para a execução de todo o programa de melhoria. Em um segundo estudo, Baddoo e Hall (2003) listam um conjunto de fatores desmotivadores, dos quais pode-se destacar: (1) a pressão exercida pela execução do programa de MPS; (2) as experiências negativas que os colaboradores possuem e (3) a falta de habilidade dos gerentes de projeto com as atividades do MPS.

A identificação de questões específicas que servem de indicadores da diferença entre organizações de sucesso ou não são apresentadas por Wilson et al.(2001). Estes autores verificaram que as empresas que obtiveram sucesso em programas de MPS possuem ótimos valores nas escalas referentes às perspectivas do ambiente 
organizacional onde foi aplicado o programa de MPS, quantidade de recursos disponibilizados para a condução do MPS e pessoas confiáveis como responsáveis pelo programa de melhoria. Segundo os autores, estes resultados podem ser utilizados para avaliar as condições da organização ao iniciar um programa de MPS, assim como identificar pontos relevantes que podem precisar de melhoria.

A aplicação prática de processo de software e a adoção de modelos de melhoria de processo de software são discutidas por Coleman e O'connor (2008). Os pesquisadores utilizaram o método Grounded Theory - GT (Strauss e Corbin, 1998) para análise dos dados e concluíram que os processos de software empregados na indústria irlandesa são processos proprietários e, normalmente, são adaptados ao contexto da empresa. Por fim, é observado que há certo tipo de resistência na implementação de programas de MPS, por parte dos gerentes, devido aos custos que essas atividades podem gerar, além de "aumentar a documentação e burocracia".

De maneira similar, Montoni e Rocha (2010) utilizaram GT para conduzir uma investigação de fatores de influência em programas MPS. Foram analisados os fatores socioculturais do ponto de vista dos consultores das organizações de consultoria. Segundos os autores, foi possível identificar três contextos que exercem diferentes tipos de influência no programa de MPS, são eles: Contexto individual (envolvem questões relacionadas aos membros da organização, alta gerência e da consultoria em MPS); Contexto Organizacional (questões relacionadas à estratégias e políticas organizacionais e o ambiente organizacional) e Contexto Tecnológico (relacionado ao processo de software e recursos de software e hardware que apóiam o MPS). Também foram identificadas ações que representam as interações entre os colaboradores e os diferentes contextos abordados no trabalho. No presente trabalho o foco foi direcionado ao contexto individual (especificamente membros da organização), buscando detalhar as influências humanas em um programa de MPS.

A pesquisa desenvolvida neste trabalho procura aprofundar a compreensão sobre os aspectos humanos que influenciam um programa de MPS. No entanto, aspectos humanos não são coletados com facilidade, o que dificultou sua coleta nas etapas iniciais da pesquisa. $\mathrm{O}$ entendimento destes fatores pode contribuir para uma eficiente institucionalização dos processos de software, compreender determinadas reações dos colaboradores que criaram uma resistência a um programa de MPS e outros fatores relacionados à MPS.

\section{A Condução da Pesquisa Qualitativa}

O principal objetivo desta pesquisa é identificar os aspectos humanos que influenciam um programa de MPS com a finalidade de obter uma maior compreensão desses fatores para um programa de melhoria. $\mathrm{O}$ contexto desta pesquisa consiste em três empresas participantes do pólo de software do Amazonas, especificamente as três primeiras empresas avaliadas no programa MPS.BR. Para esta pesquisa, colaboradores que participaram dos projetos avaliados no programa de MPS em diferentes papéis (gerentes, analistas e desenvolvedores) foram entrevistados. Desta forma, pôde-se investigar o ponto de vista do colaborador a partir de suas afirmações e compreender os fatores que, segundo estes colaboradores, influenciaram o programa de melhoria. 
Esta pesquisa foi conduzida em três diferentes fases: antes da avaliação oficial MPS.BR, logo após a avaliação e cerca de quatro meses após. Em cada fase da pesquisa foi realizada uma sequência de coleta e análise dos dados. Sendo esta uma pesquisa de cunho qualitativo, foram adotados métodos apropriados, descritos a seguir.

\subsection{Métodos de Coleta e Análise de Dados Qualitativos}

Para realizar a coleta de dados optou-se por utilizar entrevistas semi-estruturadas baseadas em questões semi-abertas, pois segundo Yin (2001), "Entrevistas semiestruturadas tem como objetivo principal compreender os significados que os entrevistados atribuem às questões e situações relativas aos temas de interesse".

A análise dos dados qualitativos foi feita com base nos procedimentos do método Grounded Theory - GT (ou Teoria Fundamentada em Dados) (Strauss e Corbin, 1998). O método GT descreve um conjunto de procedimentos sistemáticos de coleta e análise dos dados para gerar, elaborar e validar teorias substantivas sobre fenômenos essencialmente sociais, ou processos sociais abrangentes (Bandeira-De-Mello e Cunha, 2003). O método foi escolhido devido à escassez de pesquisas identificadas que tratam aspectos humanos em implantações de MPS e ao fato de que a pesquisa ter procurado identificar características humanas de uma maneira ampla, possibilitando assim encontrar aspectos que não foram destacados em pesquisas anteriores.

Especificamente, para a análise dos dados foram utilizados procedimentos de codificação, que é o processo sugerido pela GT como mecanismo de análise dos dados. Durante a codificação são identificados conceitos (ou códigos) e categorias. Um conceito dá nome a um fenômeno de interesse para o pesquisador; abstrai um evento, objeto, ação, ou interação que tem um significado para o mesmo (Strauss e Corbin, 1998). Categorias são agrupamentos de conceitos em um grau de abstração mais alto.

O processo de codificação é dividido em três fases: codificação aberta (envolve a quebra, a análise, a comparação, a conceituação e a categorização dos dados), codificação axial (explicitam-se as relações entre os códigos, mostrando relações de causas e efeitos, condições intervenientes e estratégias de ação) e codificação seletiva (fase que integra todas as outras categorias e expressar a essência do processo social que ocorre entre os envolvidos).

Os métodos de coleta e análise de dados foram utilizados nas três diferentes fases realizadas nesta pesquisa. A seguir é descrito o processo seguido em cada um destas fases.

\subsection{Processo seguido nos Estudos Qualitativos}

O processo seguindo em cada fase desta pesquisa é apresentado na Figura 1 e consiste de três etapas: (1) Planejamento, (2) Execução e Análise e (3) Avaliação dos Resultados.

Na etapa de Planejamento realizou-se a atividade de definição do escopo da fase da pesquisa qualitativa. Nesta atividade definiu-se o que seria pesquisado e o que se esperava de resultados. Em seguida, foram preparados os instrumentos de coleta e análise de dados. Nesta atividade, definiram-se as questões do questionário que foram utilizadas nas entrevistas. Os questionários das primeiras etapas foram desenvolvidos 
com base em estudos de fatores críticos na implantação de MPS. Na última etapa de pesquisa empírica foi utilizado um modelo mais abrangente que estuda aspectos humanos nas organizações. Em todas as etapas, o instrumento de pesquisa foi revisado por diferentes pesquisadores.

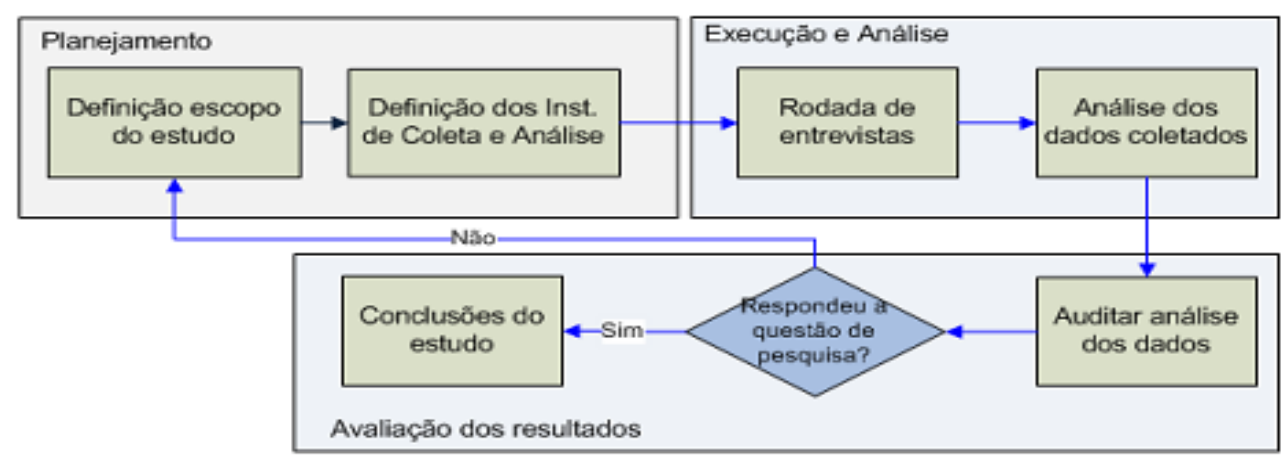

Figura 1 - Processo seguido em cada fase da pesquisa.

A etapa de Execução e Análise possuiu duas atividades. A primeira atividade foi a condução das entrevistas nas empresas. Em cada fase da pesquisa, os colaboradores assinaram um termo sobre a confidencialidade das informações fornecidas pelos colaboradores. Em seguida, as entrevistas foram transcritas na íntegra para serem utilizadas na análise dos dados. A segunda atividade corresponde à análise dos dados, esta atividade foi executada seguindo os procedimentos metodológicos da GT.

A primeira fase do método GT (codificação aberta) corresponde à criação de códigos que estão relacionados a trechos do texto referentes aos aspectos humanos, conforme exemplificado na Figura 2. Várias interações de comparações foram realizadas para a seleção de códigos que indicavam relatos representativos em citações no texto.

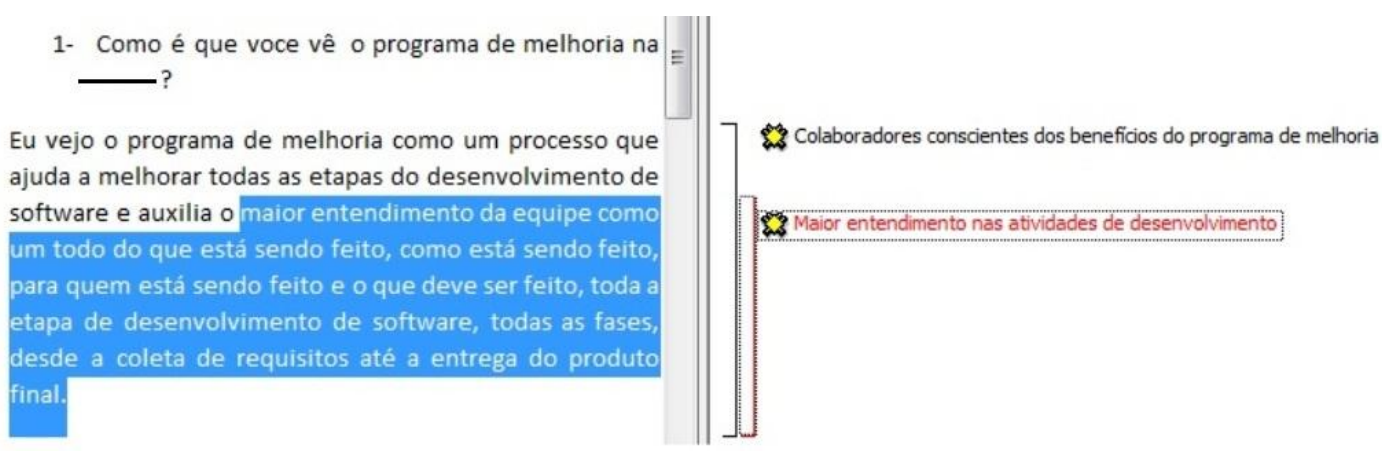

Figura 2 - Trecho de uma entrevista: do lado esquerdo está o documento com as citações e à direita os códigos criados a partir da citação identificada.

Ainda na atividade de análise, iniciou-se a fase de codificação axial, onde foram criados os relacionamentos entre códigos, gerando inter-relações que agrupam a natureza dos fatores envolvidos em programas de MPS. A Figura 3 apresenta um exemplo da codificação axial. 
$\mathrm{Na}$ etapa de avaliação dos resultados, os códigos e os relacionamentos foram analisados em conjunto de outros pesquisadores envolvidos na pesquisa, de forma a auditar as análises realizadas. Esta estratégia foi adotada para assegurar o rigor da aplicação dos métodos qualitativos. Se os dados encontrados respondessem a questão de pesquisa, os dados eram formatados em conclusões. Se os dados não fossem satisfatórios, era necessário fazer um novo planejamento e iniciar uma nova fase. $\mathrm{Na}$ atividade de conclusão do estudo, os relacionamentos entre os códigos foram analisados criando as proposições sobre as influências encontradas nas fases do estudo qualitativo.

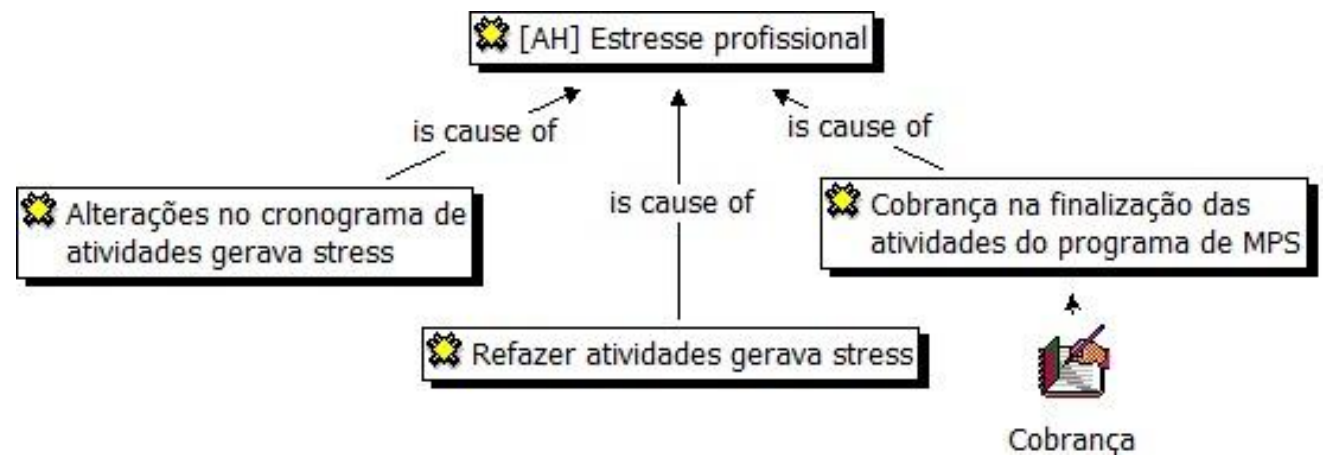

Figura 3 - Representação gráfica da codificação axial

\section{Resultados das fases da Pesquisa}

Conforme explicado previamente, nesta pesquisa três fases de coleta e análise de dados foram realizadas em diferentes momentos do programa de melhoria com diferentes instrumentos de coleta. A primeira e a segunda fases desta pesquisa foram descritas detalhadamente em publicações anteriores (Matos et al., 2010; Santos et al., 2010). Por esta razão, este artigo não objetiva descrever como cada fase foi realizada e sim discutir seus resultados. Assim, os resultados de cada fase da pesquisa são apresentados a seguir.

\subsection{Primeira Fase: durante a impementação do MPS}

A primeira fase foi realizada durante a implementação do programa de melhoria nas empresas A e B, isto é, antes da avaliação oficial MPS.BR. Quatro colaboradores, que estavam diretamente envolvidos com a implementação do programa de melhoria, foram entrevistados.

Embora o objetivo deste estudo inicial fosse identificar a influência dos aspectos humanos naquele momento do programa de MPS, as questões semi-abertas formuladas nas entrevistas levaram à identificação de diferentes aspectos que estavam influenciando o MPS, além de impactos que o mesmo trouxe para as organizações. As principais categorias e fatores de influência encontrados nesta fase são apresentadas na Tabela 1.

Tabela 1. Resumo dos resultados do primeiro estudo

\begin{tabular}{|c|c|c|}
\hline $\begin{array}{c}\text { Tipos de } \\
\text { Fatores }\end{array}$ & Fatores & \multicolumn{2}{|c|}{ Resultados Encontrados } \\
\hline$++-\infty \cdot-$ & Comprometimento da & Colaboradores empenhados em obter a "certificação". Alta \\
\hline
\end{tabular}




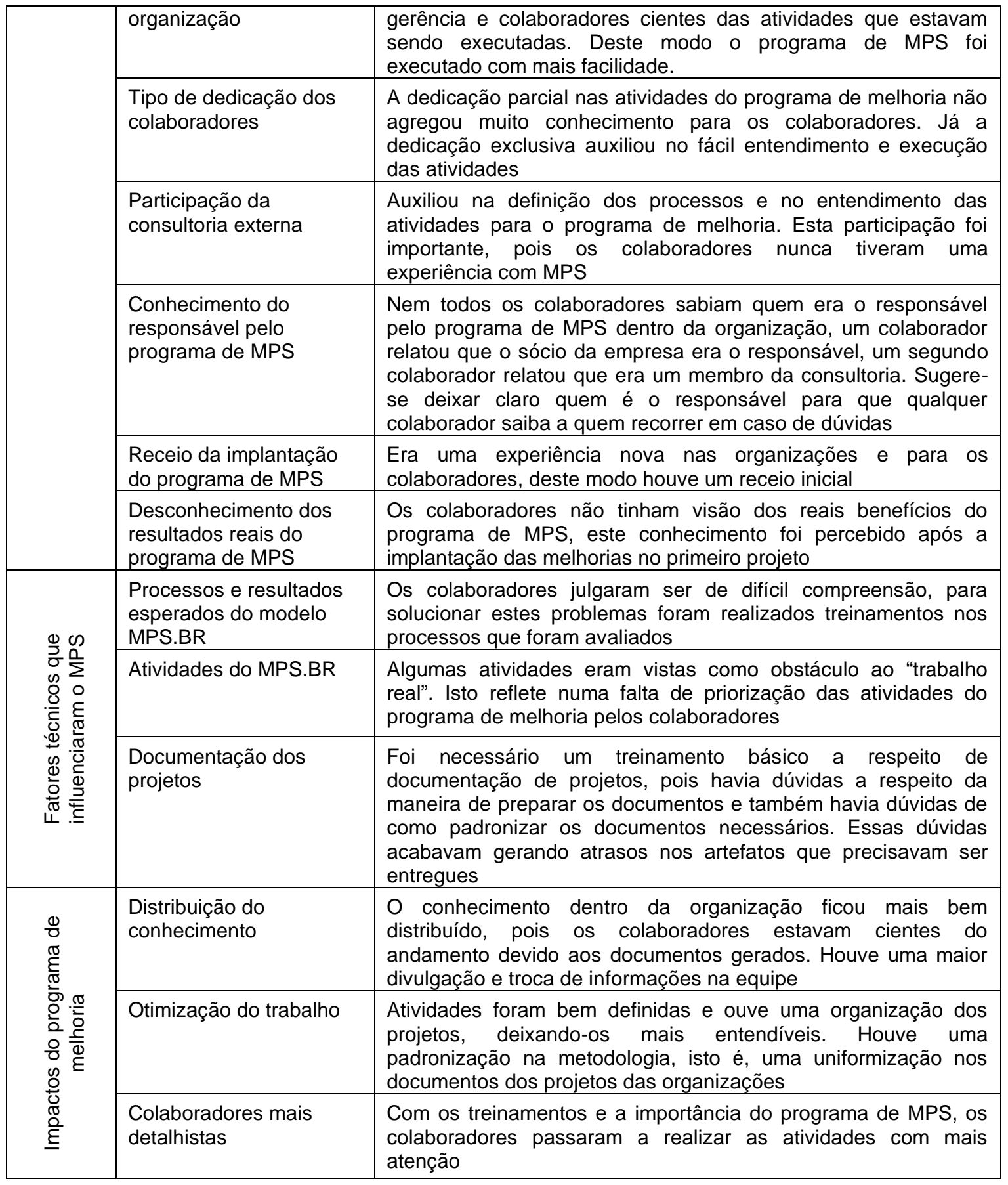

Através da análise dos dados foi possível verificar diferentes aspectos que influenciaram o programa de MPS e alguns indícios a respeito de aspectos humanos, como por exemplo, a motivação da equipe em conseguir a "certificação". No entanto, o instrumento de coleta de dados utilizado (perguntas semi-estruturadas do questionário) não possibilitou um destaque relativo aos aspectos humanos. Desta forma, foi necessário rever este instrumento para obter um maior aprofundamento sobre os aspectos humanos.

\subsection{Segunda Fase: Após a avaliação MPS.BR}

A segunda fase da pesquisa qualitativa ocorreu após a avaliação oficial do modelo de melhoria. Três colaboradores (empresa $\mathrm{C}$ ) que participaram ativamente do programa de 
MPS foram entrevistados. Desta forma, foi possível obter informações a respeito de dois momentos importantes do programa de melhoria.

Como os resultados obtidos na primeira fase não abrangiam de maneira satisfatória os aspectos humanos, um novo planejamento do estudo, principalmente nos instrumentos de coleta foi necessário. Essas alterações visavam à captura mais precisa de informações sobre os aspectos humanos que poderiam influenciar um programa de melhoria de processo de software. Percebeu-se que neste momento os colaboradores se sentiam mais confortáveis a falar, pois já havia passado a avaliação do MPS de suas organizações. Um resumo dos resultados desta segunda fase é apresentado na Tabela 2.

Tabela 2. Resumo dos resultados do segundo estudo

\begin{tabular}{|c|c|c|}
\hline $\begin{array}{l}\text { Tipos de } \\
\text { Fatores }\end{array}$ & Fatores & Resultados Encontrados \\
\hline \multirow{5}{*}{ 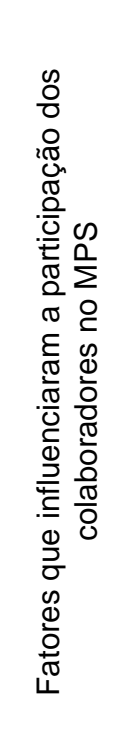 } & $\begin{array}{l}\text { Comprometimento } \\
\text { dos colaboradores }\end{array}$ & $\begin{array}{l}\text { Os colaboradores viam o programa de MPS como uma oportunidade } \\
\text { de obter diferencial profissional, Logo, eles se comprometeram com } \\
\text { as atividades necessárias para implementar o programa de melhoria. } \\
\text { Devido ao contexto das empresas (pequenas empresas e afiliadas a } \\
\text { incubadoras) o programa de MPS se tornou um desafio para os } \\
\text { colaboradores. }\end{array}$ \\
\hline & $\begin{array}{l}\text { Tipo de dedicação } \\
\text { dos colaboradores }\end{array}$ & $\begin{array}{l}\text { Assim como na primeira fase, verificou-se que a dedicação exclusiva } \\
\text { de alguns colaboradores foi importante para o sucesso do MPS. }\end{array}$ \\
\hline & $\begin{array}{l}\text { Resistência ao } \\
\text { programa de MPS }\end{array}$ & $\begin{array}{l}\text { Os colaboradores julgavam que as atividades do programa de MPS } \\
\text { eram trabalhosas e complicadas isto fazia com que não tivessem } \\
\text { muita motivação para realizar as atividades necessárias. }\end{array}$ \\
\hline & $\begin{array}{l}\text { Conhecimento } \\
\text { anterior ao } \\
\text { programa de MPS }\end{array}$ & $\begin{array}{l}\text { Alguns colaboradores julgaram ter conhecimentos em gerência de } \\
\text { projetos e isto auxiliou na definição e execução das atividades do } \\
\text { programa de MPS. }\end{array}$ \\
\hline & $\begin{array}{l}\text { Decisões a respeito } \\
\text { do programa de } \\
\text { MPS }\end{array}$ & $\begin{array}{l}\text { Algumas decisões da diretoria desagradavam alguns colaboradores } \\
\text { envolvidos com o programa de melhoria, como a definição estratégica } \\
\text { dos projetos/clientes utilizados para ser apresentados na avaliação. }\end{array}$ \\
\hline \multirow{4}{*}{ 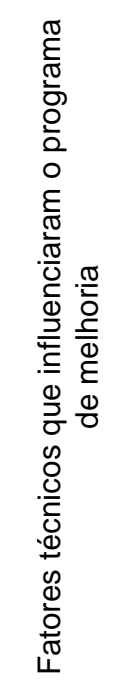 } & $\begin{array}{l}\text { Padronização do } \\
\text { processo }\end{array}$ & $\begin{array}{l}\text { Uma vez bem definida a documentação e as atividades necessárias, } \\
\text { foi possível padronizar o processo da organização. Esta padronização } \\
\text { do processo possibilitou a visualização mais completa dos objetivos a } \\
\text { serem alcançados nos projetos de software. Passou-se a ter uma } \\
\text { maior dimensão do tamanho dos projetos da organização. }\end{array}$ \\
\hline & $\begin{array}{l}\text { Organização dos } \\
\text { artefatos }\end{array}$ & $\begin{array}{l}\text { Houve melhoria na comunicação interna da equipe e na formalização } \\
\text { da interação com o cliente, desta maneira é possível ter evidências de } \\
\text { tudo que foi tratado junto ao cliente. Colaboradores reportaram } \\
\text { também que houve uma melhoria na qualidade do serviço prestado. }\end{array}$ \\
\hline & Treinamento & $\begin{array}{l}\text { Devido às dificuldades encontradas, foi necessário prover } \\
\text { treinamentos para os colaboradores. Assim como na primeira fase, foi } \\
\text { observado que alguns tinham dificuldade em questões básicas de } \\
\text { engenharia de software. }\end{array}$ \\
\hline & $\begin{array}{l}\text { Falta de priorização } \\
\text { para as atividades } \\
\text { do programa de } \\
\text { MPS }\end{array}$ & $\begin{array}{l}\text { Os colaboradores também executam as atividades do dia-a-dia da } \\
\text { empresa, desta forma algumas atividades do programa de melhoria se } \\
\text { tornavam secundárias, como por exemplo, atender uma solicitação de } \\
\text { mudança de requisito do cliente sem antes realizar todo o registro de } \\
\text { solicitação. }\end{array}$ \\
\hline
\end{tabular}

$\mathrm{Na}$ etapa de avaliação desta segunda fase, conclui-se que os dados ainda não respondiam a questão de pesquisa, sendo necessário novo estudo, apresentado a seguir, onde o questionário foi revisto e foram incluídas questões com foco em fatores humanos. 


\subsection{Terceira Fase: Aprofundamento da Pesquisa sobre Fatores Humanos}

Para adequar a criação e análise das categorias e propriedades relevantes para o objeto de estudo desta pesquisa, decidiu-se realizar uma revisão da literatura sobre aspectos humanos, de forma a aumentar o enfoque das questões das entrevistas nesses aspectos. Desta forma, foi necessário analisar e reformular os instrumentos de coleta com a finalidade de adequá-los ainda mais a pesquisa.

Uma revisão dos aspectos humanos advindos do comportamento organizacional, descrito em Robbins (2005), foi realizada. Esta revisão foi necessária devido à complexidade dos aspectos humanos que possuem diversas definições na literatura. Desta forma, foi preciso definir os conceitos de aspectos humanos considerados nesta pesquisa. A Tabela 3 apresenta os aspectos relevantes no contexto desta pesquisa.

Tabela 3. Conceitos dos aspectos humanos considerados nesta pesquisa

\begin{tabular}{|c|c|}
\hline Aprendizagem & $\begin{array}{l}\text { Qualquer mudança relativamente permanente no comportamento de um } \\
\text { indivíduo. Logo, a mudança no comportamento determina se houve } \\
\text { aprendizagem com a experiência em determinada atividade. }\end{array}$ \\
\hline Motivação & $\begin{array}{l}\text { Processo responsável pela intensidade, direção e persistência dos esforços de } \\
\text { uma pessoa para o alcance de uma determinada meta. }\end{array}$ \\
\hline Personalidade & $\begin{array}{l}\text { Organização dinâmica interna dos sistemas psicológicos do indivíduo que } \\
\text { determinam seu ajuste individual ao ambiente. Soma das maneiras como uma } \\
\text { pessoa reage e interage com as demais. }\end{array}$ \\
\hline Emoções & $\begin{array}{l}\text { Sentimentos intensos direcionados a alguém ou a algo. São reações a um objeto } \\
\text { e não um traço. Você mostra suas emoções quando está "feliz com alguma coisa, } \\
\text { bravo com alguém ou com medo de algo". }\end{array}$ \\
\hline Percepção & $\begin{array}{l}\text { Baseia-se na interpretação que as pessoas fazem da realidade e não na } \\
\text { realidade em si. Processo pelo qual os indivíduos interpretam suas impressões } \\
\text { sensoriais com a finalidade de dar sentido ao ambiente. }\end{array}$ \\
\hline Treinamento & $\begin{array}{l}\text { Processo que visa melhorar o desenvolvimento profissional do ser humano na } \\
\text { sua organização e no desempenho das suas funções além de ser o processo que } \\
\text { visa à preparação e ao aperfeiçoamento das habilidades e dos conhecimentos } \\
\text { dos funcionários. }\end{array}$ \\
\hline $\begin{array}{l}\text { Eficácia de } \\
\text { Liderança }\end{array}$ & $\begin{array}{l}\text { Liderança é uma influência interpessoal exercida em uma dada situação para } \\
\text { consecução de um ou mais objetivos. }\end{array}$ \\
\hline $\begin{array}{l}\text { Satisfação com o } \\
\text { trabalho }\end{array}$ & Refere-se à atitude geral de uma pessoa em relação ao trabalho que ela realiza. \\
\hline $\begin{array}{l}\text { Tomada de } \\
\text { decisão individual }\end{array}$ & $\begin{array}{l}\text { Associado a escolher entre duas ou mais alternativas: (1) ocorre em reação a um } \\
\text { problema ou (2) a todas as decisões requerem interpretação e avaliação das } \\
\text { informações. }\end{array}$ \\
\hline $\begin{array}{l}\text { Avaliação de } \\
\text { desempenho }\end{array}$ & $\begin{array}{l}\text { Verifica resultados individuais das tarefas, utilizando variáveis de produtividade } \\
\text { do indivíduo para mensurar seu desempenho. }\end{array}$ \\
\hline $\begin{array}{l}\text { Mensuração de } \\
\text { atitudes }\end{array}$ & $\begin{array}{l}\text { Verificada pelas das ações dos colaboradores dentro das organizações que } \\
\text { refletem como os mesmos se sentem em relação a algo. }\end{array}$ \\
\hline $\begin{array}{l}\text { Seleção de } \\
\text { pessoal }\end{array}$ & Processo de adequar às características individuais aos requisitos do trabalho. \\
\hline $\begin{array}{l}\text { Planejamento de } \\
\text { trabalho }\end{array}$ & $\begin{array}{l}\text { E necessário planejar todas as atividades para que os indivíduos possam } \\
\text { trabalhar e assumir responsabilidades. }\end{array}$ \\
\hline $\begin{array}{l}\text { Estresse } \\
\text { profissional }\end{array}$ & $\begin{array}{l}\text { Condição dinâmica na qual o indivíduo é confrontado com uma oportunidade, } \\
\text { limitação ou demanda em relação a alguma coisa referente ao trabalho. }\end{array}$ \\
\hline
\end{tabular}

Inicialmente realizou-se uma entrevista-piloto com um colaborador da organização $\mathrm{C}$ com a finalidade de verificar a efetividade do instrumento de coleta de dados. Uma vez verificado que o questionário poderia capturar dados relevantes, deu-se prosseguimento à pesquisa. 
Optou-se por fazer a análise em separado das organizações a fim de melhor verificar o surgimento dos códigos e categorias, além de verificar as diferenças de achados nas duas organizações. Nesta análise foram realizadas três entrevistas na organização A, cuja análise está descrita detalhadamente em Santos et al. (2011), e duas entrevistas na organização B. As entrevistas foram realizadas quatro meses após a segunda fase da pesquisa.

Os resultados apresentados são referentes aos aspectos em que foi possível encontrar achados mais relevantes para a pesquisa. A seguir será apresentada uma comparação dos resultados encontrados nas duas organizações.

$\mathrm{O}$ aspecto humano relacionado à aprendizagem foi verificado nas duas organizações. Porém, na organização A observou-se que o processo de aprendizagem das atividades do programa de MPS gerava um atraso na entrega dos produtos de trabalho, pois era necessário tempo para o aprendizado. Já na organização $B$, foi relatado que a aprendizagem ocorreu quando os conhecimentos adquiridos eram postos em prática. Em relação aos colaboradores que não participaram ativamente do programa de MPS, porém acompanharam a implementação, verificou-se que poucos assimilaram os conhecimentos de gerência de projetos e gerência de requisitos. Nas duas organizações observou-se que o conhecimento anterior (sobre processo de software e Gerência de Projetos) auxiliou numa melhor aprendizagem das atividades do programa de MPS.

Em relação ao fator motivação, verificou-se que diversos fatores motivaram os colaboradores na organização A, destacando-se: (1) a organização dos artefatos de trabalho dos projetos, pois era possível ter respaldo em relação a possíveis reclamações dos clientes; (2) obtenção de novos conhecimentos em engenharia de software (que também motivou a organização B); (3) conseguir o nível desejado na avaliação do modelo MPS (que também foi percebido na organização B); (4) ser a primeira empresa avaliada no modelo de maturidade no Estado do Amazonas. Na organização B, Além dos já mencionados como similares na organização, os colaboradores se sentiam motivados quando eram realizadas atividades de integração dos colaboradores.

Ainda em relação à motivação, foram identificados alguns fatores que faziam com que a alta gerência não conseguisse motivar os colaboradores, na organização A, tem-se : (1) falta de um sistema de recompensa pelas atividades realizadas e (2) falta de reconhecimento dos colaboradores que participaram ativamente do programa de MPS. Já na organização B, destacam-se: (1) a visão de obrigatoriedade que os colaboradores tinham em relação às atividades do programa de melhoria; (2) a falta de controle por parte da organização, pois era necessário verificar com mais detalhes a execução todas as atividades do programa de MPS. Deste modo, uma das lições deste estudo é que estes fatores sejam verificados nas próximas implementações a fim de não comprometer $o$ sucesso do MPS.

Ao se verificar o aspecto humano relacionado à satisfação do trabalho, na organização A verificou-se que há divergência na satisfação em trabalhar em um programa de MPS, segundo os entrevistados nem todos os colaboradores se sentiam satisfeitos em trabalhar no programa de melhoria. Em contrapartida, verificou-se que a satisfação apontada pelos colaboradores diz respeito à flexibilidade de horário na época da implantação do programa de melhoria. Uma vez perguntado se gostariam de se envolver novamente em um programa de melhoria dentro da organização, os 
colaboradores afirmaram que sim, o que demonstra a satisfação em trabalhar com o programa de melhoria de processo de software. Já na organização B foi identificado que havia satisfação em trabalhar em programa de melhoria, pois os colaboradores tinham foco nas atividades e buscavam resultado no programa de MPS. Porém, apesar da satisfação, os colaboradores julgaram que as atividades eram trabalhosas.

Entre os achados relacionados à personalidade, percebeu-se na organização A que a pró-atividade foi um traço que auxiliou a resolver problemas durante o programa de melhoria. Já na organização $B$, verificou-se a capacidade de motivar outros colaboradores e o sentimento de ser o exemplo para os demais colaboradores.

O julgamento em relação à eficácia da liderança foi verificado com a conclusão da implementação do processo de software e o alcance do nível $G$ na avaliação MPS.BR. Colaboradores das duas organizações avaliaram que houve uma liderança efetiva no programa de MPS, pois haviam pessoas capacitadas executando as atividades necessárias. Verificou-se que a dedicação exclusiva para executar as atividades do programa de melhoria influenciou na liderança efetiva nas duas organizações.

Quando era necessário tomar uma decisão, os colaboradores das duas organizações tomavam decisões de forma consciente, pois: (1) eles analisavam os motivos dos eventos para tomar uma decisão, (2) A alta gerência normalmente era focada em gerenciar o programa de MPS, (3) As decisões a serem tomadas eram discutidas antes de serem repassadas para os demais colaboradores. Porém quando era necessário atender algum cliente, a decisão automaticamente já estava tomada: o cliente era prioridade.

Em relação à avaliação de desempenho, observou-se que os colaboradores das duas organizações tiveram um bom desempenho nas atividades do programa de MPS, pois fizeram tudo que era necessário para conseguir o nível esperado. Porém na organização A, verificou-se que nem todos os colaboradores tiveram um nível de desempenho similar.

Analisando o aspecto estresse profissional, notou-se algumas causas que levaram ao estresse dos colaboradores: (1) alteração no cronograma das atividades do programa de melhoria; (2) refazer algumas atividades; (3) cobrança na finalização das atividades. Vale ressaltar que somente a terceira causa foi encontrada na organização B e A. As demais foram verificadas somente na organização A. Adicionalmente, foi verificado que a demora para entregar os produtos de software também estava relacionada ao estresse.

O desenvolvimento profissional dos colaboradores através dos treinamentos foi auxiliado pela consultoria externa que os treinou em conceitos e atividades processos que seriam avaliados: gerência de projetos e gerência de requisitos, deste modo os colaboradores sabiam o que tinham que fazer e como tinham que realizar as atividades requeridas pelo programa de melhoria. Os treinamentos possibilitaram aos colaboradores o conhecimento sobre quem era o responsável pelo programa de MPS e o seu papel na condução deste programa.

Finalmente, em relação ao aspecto percepção, os colaboradores estavam conscientes em relação ao andamento dos projetos, uma vez que tinha todo o processo definido, isto mostra que os mesmos tinham ciência das atividades executadas dentro da organização. 
Os aspectos humanos apresentados nesta pesquisa devem ser interpretados de forma cuidadosa para possibilitar um auxílio em futuras implementações de programas de MPS. A seguir é apresentado um resumo sobre os achados desta pesquisa.

\subsection{Sugestões de práticas resultantes da pesquisa}

A Tabela 4 apresenta um conjunto de sugestões que outros profissionais devem aproveitar em futuros programas de melhoria. Essas sugestões são baseadas nos resultados desta pesquisa qualitativa.

Tabela 4. Sugestões a partir dos resultados da pesquisa

\begin{tabular}{|c|c|}
\hline Aprendizagem & $\begin{array}{l}\text { É fundamental verificar o conhecimento anterior dos colaboradores nos conceitos } \\
\text { dos processos que serão implementados (no caso desta pesquisa Gerência de } \\
\text { Projetos e Gerência de Requisitos) para criar direcionamentos adequados a fim } \\
\text { de que os mesmos aprendam as atividades do programa de MPS de forma eficaz. } \\
\text { Sugere-se fazer com que todos os colaboradores pratiquem o que foi aprendido } \\
\text { em projetos-piloto ou pelo menos em sala de aula. Com isto é possível } \\
\text { institucionalizar os processos dentro da organização. }\end{array}$ \\
\hline Treinamento & $\begin{array}{l}\text { Com os treinamentos é necessário verificar se os colaboradores realmente } \\
\text { sabem o que fazer e como fazer. O treinamento também deve auxiliar na } \\
\text { definição dos papeis dentro da organização. }\end{array}$ \\
\hline Motivação & $\begin{array}{l}\text { Aconselha-se criar meios para motivar os colaboradores em relação às atividades } \\
\text { do programa de melhoria, como: criar uma política de reconhecimento dos } \\
\text { colaboradores e um sistema de recompensas. É indicado também apresentar a } \\
\text { real importância do programa de melhoria e explicar sobre a importância dos } \\
\text { prazos, tentando evitar que isso se torne uma pressão nos colaboradores. }\end{array}$ \\
\hline Personalidade & $\begin{array}{l}\text { É relevante identificar colaboradores chaves, ou seja, colaboradores que sejam } \\
\text { pró-ativos e que consigam motivar outros colaboradores a fim de que eles } \\
\text { possam auxiliar na disseminação do programa de MPS. }\end{array}$ \\
\hline Percepção & $\begin{array}{l}\text { É importante que tanto os colaboradores quanto a alta gerência tenha percepção } \\
\text { do programa de MPS. Deste modo, ela pode estar informada sobre o que está } \\
\text { acontecendo em relação às atividades do programa de melhoria na organização. }\end{array}$ \\
\hline $\begin{array}{l}\text { Eficácia de } \\
\text { Liderança }\end{array}$ & $\begin{array}{l}\text { É necessário verificar a capacidade de liderança do responsável pelo programa } \\
\text { de MPS na organização sob pena de não se obter êxito na implementação do } \\
\text { MPS. Notou-se também que a dedicação exclusiva do responsável pelo MPS foi } \\
\text { importante para a execução das atividades. }\end{array}$ \\
\hline $\begin{array}{l}\text { Satisfação com o } \\
\text { trabalho }\end{array}$ & $\begin{array}{l}\text { A satisfação com as atividades do programa de MPS é obtida através de } \\
\text { benefícios ao colaborador, como flexibilidade no horário de trabalho. }\end{array}$ \\
\hline $\begin{array}{l}\text { Tomada de } \\
\text { decisão individual }\end{array}$ & $\begin{array}{l}\text { As decisões devem tomadas de forma consciente, os motivos precisam ser } \\
\text { analisados e discutidos com os responsáveis pelo programa de melhoria, antes } \\
\text { de ser repassados para os demais colaboradores. }\end{array}$ \\
\hline $\begin{array}{l}\text { Avaliação de } \\
\text { desempenho }\end{array}$ & $\begin{array}{l}\text { É sugerido fazer uma avaliação entre os colaboradores a fim de identificar os } \\
\text { pontos de melhoria, pois diferentes níveis de desempenho podem comprometer o } \\
\text { programa de melhoria. }\end{array}$ \\
\hline $\begin{array}{l}\text { Estresse } \\
\text { profissional }\end{array}$ & $\begin{array}{l}\text { Deve-se ter cautela com eventos que ocorrem na organização como, por } \\
\text { exemplo, na cobrança da finalização das atividades e no retrabalho de } \\
\text { determinadas atividades do programa de melhoria. }\end{array}$ \\
\hline
\end{tabular}

\section{Conclusões e trabalhos futuros}

Esse trabalho descreveu os resultados de uma pesquisa qualitativa com a finalidade de compreender os aspectos humanos em um programa de MPS. Para a realização deste trabalho, foram conduzidas entrevistas em organizações de software pioneiras na implementação do nível G do MPS.BR no Estado do Amazonas.

Neste trabalho foi possível identificar e compreender alguns aspectos que influenciaram a implementação do programa de MPS nas empresas estudadas. Verificaram-se questões importantes a respeito do processo de aprendizagem, treinamentos, motivação, satisfação com o trabalho, personalidade, eficácia da 
liderança, tomada de decisões, avaliação de desempenho, estresse profissional e percepção do programa de MPS. Deste modo, uma das lições desta pesquisa é a necessidade de um planejamento destes aspectos humanos nas próximas implementações a fim de não comprometer o sucesso do MPS.

Uma possível extensão deste trabalho é replicar a pesquisa realizada nos mais diversos programas de MPS do país com a finalidade de identificar similaridades dos aspectos humanos que influenciam estes programas. Além disso, nem todos os aspectos humanos identificados por Robbins (2005) foram percebidos como significativos pelos entrevistados, desta forma, novas pesquisas poderiam aprofundar especificamente estes aspectos visando avaliar se, realmente, os mesmos não são influentes em programas de MPS.

Como trabalhos futuros, uma comparação com o que já foi abordado na literatura será feita com a finalidade de encontrar similaridades nos estudos já realizados anteriormente por outros pesquisadores.

\section{Agradecimentos}

Os autores agradecem aos colaboradores das organizações entrevistadas. Agradecemos também o apoio financeiro do CNPq. O terceiro autor agradece ao CNPq (Edital Universal 473220/2008-3) e à FAPESPA (Edital Universal N 003/2008).

\section{Referências}

Baddoo, N., Hall, T., 2002, "Motivators of Software Process Improvement: an analysis of practitioners' views", Journal of Systems and Software, v. 62, n. 2, pp. 85-96.

Baddoo, N., Hall, T., 2003, "De-motivators for software process improvement: an analysis of practitioners' views", Journal of Systems and Software, v. 66, n. 1, pp. 23-33.

Bandeira-De-Mello, R., Cunha, C., 2003, "Operacionalizando o método da Grounded Theory nas Pesquisas em Estratégia: técnicas e procedimentos de análise com apoio do software ATLAS/TI", Curitiba, Brazil.

Coleman, G., O'connor, R., 2008, "Investigating software process in practice: A grounded theory perspective", Journal of Systems and Software, v. 81, pp. 772-784.

Conte, T., Cabral, R., Travassos, G.H., 2009, "Aplicando Grounded Theory na Análise Qualitativa de um Estudo de Observação em Engenharia de Software - Um Relato de Experiência". In: V WOSES 2009 v. 1, pp. 26 - 37, Ouro Preto, MG, Brasil.

Dybå, T., 2000, "An Instrument for Measuring the Key Factors of Success in Software Process Improvement", Journal of Empirical Software Engineering, v. 4, pp. 357-390.

Dybå, T., 2003, "Factors of software process improvement success in small and large organizations: an empirical study in the scandinavian context", Proceedings of the 9th European software engineering conference, Helsinki, Finland.

Fernandes, P.G., Oliveira, J.L., 2010, "Perfil Cultural e Institucionalização de Processos de Software: Estudo de Caso em Duas Organizações de Software". In: VI WOSES 2010 Belém, PA - Brasil.

Godoi, C.K., Bandeira-De-Melo, R., Silva, A.B.D., 2006, "Pesquisa Qualitativa e o debate sobre a propriedade de pesquisar". In: GODOI, C.K., BANDEIRA-DE-MELLO, R., SILVA, A.B.D. (eds), Pesquisa Qualitativa em Estudos Organizacionais: Paradigmas, Estratégias e Métodos, São Paulo, Saraiva.

Matos, O.O., Secatti, V.P., Santos, D.V., et al., 2010, "Aplicando Grounded Theory para Compreender os Fatores Críticos de Sucesso em Iniciativas de Melhoria de Processo de Software". In: VI WOSES 2010 Belém, PA, Brasil. 
Montoni, M., Rocha, A.R., 2010, "Aplicação de Grounded Theory para Investigar Iniciativas de Implementação de Melhorias em Processos de Software". In: IX Simpósio Brasileiro Qualidade de Software (SBQS 2010), pp. 167-181, Belém, PA.

Osterweil, L., 1987, "Software processes are software too". In: Proceedings of the 9th International conference on Software Engineering, Monterey, California, United States.

Robbins, S.P., 2005, Comportamento Organizacional, Pearson Education do Brasil Ltda.

Rocha, A.R., Montoni, M., Santos, G., et al., 2005a, "Estação TABA: Uma infra-estrutura para Implantação do Modelo de referência para Melhoria de Processo de Software". In: Simpósio Brasileiro de Qualidade de Software, pp. 49-60, Brasília.

Rocha, A.R., Montoni, M., Santos, G., et al., 2005b, "Dificuldades e Fatores de Sucesso na Implementação de Processos de Software Utilizando o MR-MPS e o CMMI", PROQUALITY (I Encontro de Implementadores de MPS.BR) (Junho), pp. 13-15.

Sales, E., Costa, A., Sales, M., et al., 2010, "WebAPSEE Pro: Um Ambiente de Apoio a Gerência de Processos de Software". In: VI Workshop Anual do MPS, Campinas.

Sandhof, K., 2004, Fatores humanos no processo de desenvolvimento de software: um estudo visando qualidade, Dissertação de M.Sc, Escola Politécnica, USP, São Paulo.

Santos, D.V., Rabelo, J., Mar, C., et al., 2010, "Resultados de um Estudo Qualitativo sobre a implementação do modelo MPS em empresas do programa AmazonSoft ". In: VI Workshop Anual do MPS (WAMPS 2010), Campinas, SP.

Santos, D.V.D., Vilela, D.C.J., Souza, C.D., et al., 2011, "Aspectos humanos que afetam um programa de melhoria de processo de software - Uma análise qualitativa". In: XIV Congresso Ibero-Americano em Engenharia de Software, Rio de Janeiro, RJ - Brasil (Artigo aceito para publicação).

Seaman, C.B., 1999, "Qualitative Methods in Empirical Studies of Software Engineering", IEEE Transactions on Software Engineering, v. 25, n. 4, pp. 557-572.

Seaman, C.B., 2008, "Qualitative Methods". In: SHULL, F., SINGER, J., SJØBERG, D.I.K., et al. (eds), Guide to Advanced Empirical Software Engineering, Springer London.

Strauss, A., Corbin, J., 1998, Basics of Qualitative Research: Techniques and Procedures for Developing Grounded Theory, 2 ed. ed. London, SAGE Publications.

Travassos, G., Kalinowski, M., 2010, "Resultados iniciais do iMPS 2010: Variação de Desempenho nas Empresas que Adotaram o Modelo MPS". In: VI Workshop Anual do MPS, pp. 24-33, Campinas, SP - Brasil.

Wilson, D.N., Hall, T., Baddoo, N., 2001, "A framework for evaluation and prediction of software process improvement success", Journal of Systems and Software, v. 59, n. 2, pp. 135-142.

Yin, R.K., 2001, "Estudo de caso: Planejamento e métodos", Porto Alegre, Bookman. 